\title{
PENTALOGÍA DE CANTRELL EN UN RECIÉN NACIDO EXPUESTO EN ÚTERO A MISOPROSTOL
}

\author{
Harry Pachajoa ${ }^{1,2}$ \\ ${ }^{1}$ Facultad de Salud, Escuela de Medicina, Universidad del Valle, Cali, Colombia. ${ }^{2}$ Grupo de Malformaciones Congénitas \\ Perinatales (MACOS).
}

\section{RESUMEN}

El misoprostol es un análogo sintético de la prostaglandina E1 y es usada por sus efectos utero-tónicos como abortivo. En los países donde se utiliza como abortivo sin éxito durante el primer trimestre de gestación, se han informado un espectro de malformaciones congénitas asociadas con la exposición prenatal, que incluyen el síndrome de Moebius, defectos de la pared abdominal, artrogriposis y anomalías por reducción de los miembros, entre otros. La pentalogía de Cantrell (PC), con una prevalencia de 1 en 65.000 nacidos vivos, es un síndrome polimalformativo que consta de 5 anomalías: un defecto de la pared abdominal, defecto esternal inferior, defecto del diafragma anterior, defecto pericárdico diafragmático y anormalidades congénitas del corazón. Se reporta un caso de pentalogía de Cantrell asociado al uso de misoprostol en el primer trimestre de la gestación, siendo este reporte el primer registro de esta asociación.

\section{PALABRAS CLAVE: Pentalogía de Cantrell, ectopia cordis, misoprostol, disrupción vascular}

\section{SUMMARY}

Misoprostol is a synthetic analogue of prostaglandin E1 and is used for effects such as uterotonic abortifacient. In countries where abortion is used as unsuccessful in the first trimester of gestation, reported a spectrum of congenital malformations associated with prenatal exposure, which include Moebius syndrome, abdominal wall defects, and anomalies as artrogriposis reduction members, among others. The pentalogy of Cantrell with a prevalence of 1 in 65,000 live births, is a syndrome consisting of 5 abnormalities: a defect in the abdominal wall, lower sternal defect, defect above the diaphragm, diaphragmatic pericardial defect, and congenital abnormalities of the heart. We report a case of Pentalogy of Cantrell associated with the use of misoprostol in the first trimester of pregnancy, this report is the first record of this association.

KEYS WORDS: Pentalogy of Cantrell, ectopia cordis, misoprostol, vascular disruption 
Misoprostol, es un análogo de la prostaglandina E1 que fue aprobado por la FDA para el tratamiento de la ulcera péptica (1). Sin embargo por ser un útero-tónico provoca contracción uterina y sangrado vaginal (2). Aunque el aborto electivo es ilegal en Colombia, este medicamento es utilizado para la interrupción del embarazo de manera clandestina y se ha considerado como un problema de salud pública (3).

En los países donde se utiliza como abortivo sin éxito durante el primer trimestre de gestación, se han informado un espectro de malformaciones congénitas asociadas con la exposición prenatal, que incluyen el síndrome de Moebius, defectos de la pared abdominal, artrogriposis y anomalías por reducción de los miembros, entre otros $(4,5,6)$. Pero no se ha asociado a la pentalogía de Cantrell (PC), la característica de este patología es el onfalocele asociado a ectopia cordis. El espectro completo consiste en cinco anomalías: un defecto de la pared abdominal superior en la línea media, defecto esternal inferior, defecto del diafragma anterior, defecto del pericardio diafragmático y anormalidades congénitas del corazón (7).

El objetivo de esta comunicación es presentar un caso de pentalogía de Cantrell asociado al uso de misoprostol en el primer trimestre de la gestación, siendo este reporte, el primero según la literatura revisada.

\section{Caso clínico}

Recién nacido con PC, hijo de padres no consanguíneos, producto de embarazo de madre de 22 años, grávida 2. La ecografía obstétrica del segundo trimestre mostró un onfalocele y hernia diafragmática. No hay antecedentes de enfermedad materna aguda o crónica. La madre refirió haber utilizado misoprostol como medicamento abortivo a las 4 semanas de gestación en dosis de $400 \mathrm{mi}-$ crogramos por vía vaginal y 800 microgramos por vía oral, posterior al uso de este medicamento, la madre presenta sangrado escaso y continuó su embarazo. Se realizó cariotipo con bandeo G (700 bandas) en sangre fetal tomado a la semana 24 , el cual fue reportado: $46, X X$. En la semana 37 , se interrumpió el embarazo por cesárea con diagnóstico de onfalocele y hernia diafragmática. Se obtuvo un recién nacido vivo de sexo femenino, con talla de $50 \mathrm{~cm}$, perímetro cefálico de $35 \mathrm{~cm}$, peso: $4200 \mathrm{~g}$ y APGAR de 8 al 1 minuto y de 9 a los 5 minutos.

Se detectó en el examen físico un defecto de pared tóraco-abdominal que involucraba la región esternal inferior y la línea media de la pared abdominal hasta la zona infraumbilical, a través del defecto protruían el corazón (ectopia cordis), el hígado y las asas intestinales (Figura 1).

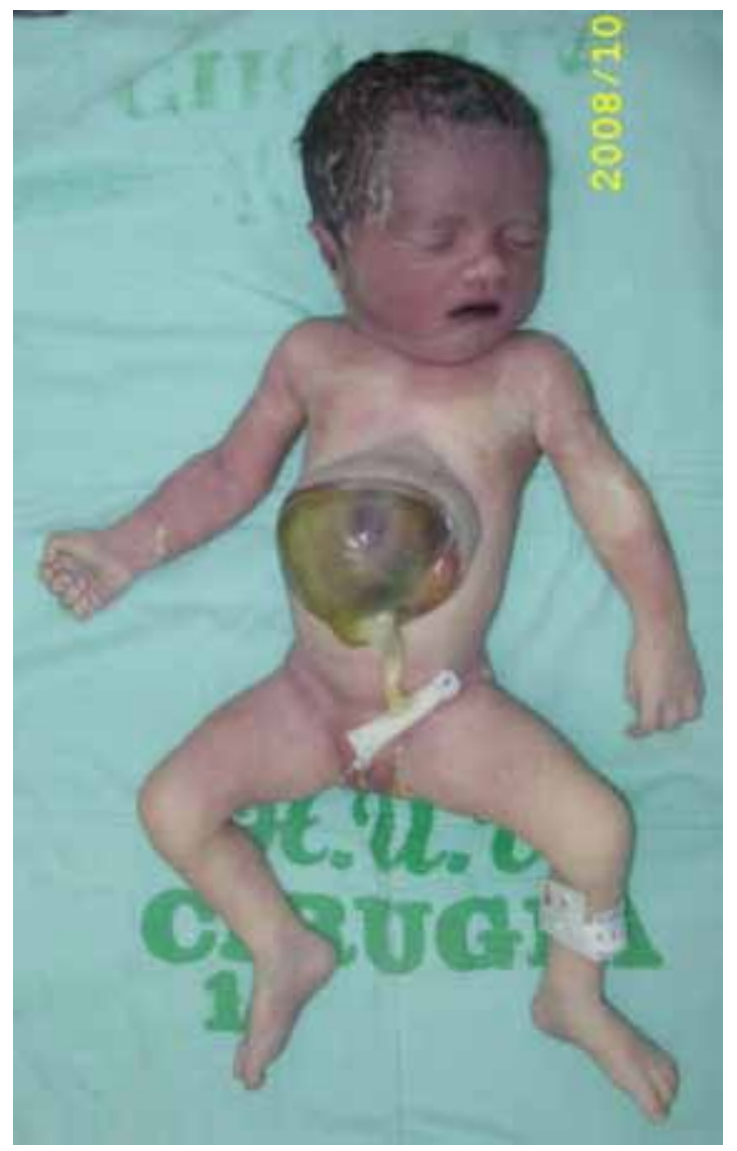

Figura 1. Recién nacida con pentalogía de Cantrell, se observa el defecto tóraco-abdominal.

En los exámenes complementarios solicitados se encontró una radiografía torácica que evidenciaba asas intestinales en el campo pulmonar izquierdo, lo que era compatible con hernia diafragmática izquierda. El ecocardiograma y la ecografía renal realizados durante el primer día de vida fueron reportados como normales. El paciente fallece a las doce horas de vida por paro cardiorespiratorio el cual fue manejado con maniobras avanzadas de reanimación. Los familiares no autorizaron la autopsia.

\section{DISCUSIÓN}

La primera descripción de la PC fue en 1958 (8). Posteriormente en 1972, Toyama sugirió la clasificación de este síndrome en: Clase 1, diagnóstico 
definido cuando se presentan los cinco defectos; Clase 2, diagnóstico probable con 4 defectos (incluyendo el intracardiaco y el defecto de pared abdominal) y Clase 3, expresión incompleta, cuando se presentan solo tres defectos (9).

La prevalencia de la PC ha sido estimada en 1 en 65.000 a 1 en 200.000 recién nacidos $(10,11)$. El caso presentado corresponde a un paciente con diagnóstico de PC clase 3 , con defecto de pared abdominal, defecto esternal inferior y hernia diafragmática, por lo que fue clasificado como de expresión incompleta.

Actualmente no es clara la etiología de este síndrome y se considera heterogénea. Hay reportes de algunos casos familiares donde se sugiere una herencia ligada al cromosoma $\mathrm{X}$, otros casos por un defecto del campo de desarrollo embrionario y en otros un defecto por disrupción vascular (12). La patogénesis de la $\mathrm{PC}$ no es clara, este defecto resulta de la falla de fusión en la línea media del mesodermo responsable de la formación de la pared tóraco-abdominal. El corazón, el hígado y las asas intestinales protruyen a través del defecto tóracoabdominal y son recubiertos por amnios (13). Sugerimos que esa falla en la fusión de la línea media puede ser originada por el misoprostol, fármaco que es reconocido como un disruptor vascular, asociación no antes reportada en la literatura revisada.

Se ha reportado la asociación de onfalocele a la exposición de misoprostol durante el primer trimestre de la gestación, aunque el mecanismo por el cuál se produce el defecto de pared no ha sido explicado por ninguno de los reportes $(4,14)$. El principal defecto asociado a la PC es el onfalocele encontrado en el $74,5 \%$ de los pacientes, defectos de esternón inferior en el $59,4 \%$, defectos diafragmáticos en el $56,8 \%$, defectos del pericardio en el $41,8 \%$ y las anomalías cardiacas en el $83 \%$ de los casos, siendo la comunicación interventricular la malformación mas reportada (11).

El diagnóstico diferencial de este defecto congénito es importante e incluye la ectopia cordis aislada y el síndrome de bandas amnióticas. El punto clave para un diagnóstico adecuado es la posición del defecto abdominal en relación con la inserción del cordón umbilical, la evisceración de los órganos, la presencia o ausencia de membranas o bandas y anormalidades asociadas (15). El defecto de pared abdominal en la PC se caracteriza por ser periumbilical, en el síndrome de bandas amnióticas se encuentran defectos o deformidades en extremidades con bandas adherentes. El pronóstico de los pacientes depende de la extensión del defecto tóraco-abdominal y de la asociación con anomalías cardiacas (16).

\section{BIBLIOGRAFÍA}

1. Norman JE, Thong KG, Baird DT. Uterine contractility and induction of abortion in early pregnancy by misoprostol and mifepristone. Lancet 1991;338:1233-6.

2. Costa SH. Commercial availability of misoprostol and induced abortion in Brazil. Int J Gynaecol Obstet 1998;63 (Suppl):131-9.

3. Isaza $\mathrm{C}$, Saldarriaga $\mathrm{W}$, Pachajoa $\mathrm{H}$. Uso indaecuado de misoprostol. ¿Un problema de salud pública? Colomb Med 2008;39(supl 2):61-5.

4. da Silva Dal Pizzol T, Knop FP, Mengue SS. Prenatal exposure to misoprostol and congenital anomalies: systematic review and meta-analysis. Reprod Toxicol 2006;22:666-71.

5. Ruiz-Botero F, Pachajoa H, Saldarriaga W, Isaza C. Artrogriposis múltiple congénita en gemelo monocoriónico biamniótico. Reporte de caso y revisión de la literatura. Rev Colomb Obstet Ginecol 2009;60(1):7982.

6. Vargas FR, Schuler-Faccini L, Brunoni D, Kim C, Meloni VFA, Sugayama SMM, et al. Prenatal exposure to misoprostol and vascular disruption defects: a casecontrol study. Am J Med Genet 2000;95:302-6.

7. Engum SA. Embryology, sternal clefts, ectopia cordis, and Cantrell's pentalogy. Sem Pediat Surgery 2008;17:154-60.

8. Cantrell JR, Haller JA, Ravitsh MM. A syndrome of congenital defects involving the abdominal wall, sternum, diaphragm, pericardium and heart. Surg Gynecol Obstet 1958;107:602-14.

9. Toyama WM. Combined congenital defects of the anterior abdominal wall, sternum, diaphragm, pericardium and heart: a case report and review of the syndrome. Pediatrics 1972;50:778-86.

10. van Allen MI, Curry C, Walden CE, Gallagher L, Patten RM. Limb-body wall complex: II. Limb and spine defects. Am J Med Genet 1987;28:549-65.

11. Vazquez-Jimenez JF, Eberhard GM, Daebritz S, Keutel J, Nishigaki K, Huegel W, Messmer BJ. Cantrell's syndrome: a challenge to the surgeon. Ann Thorac Surg 1998;65:1178-85.

12. Parvari R, Carmi R, Weissenbach J, Pilia G, Mumm S, Weinstein $Y$. Refined genetic mapping of X-linked thoracoabdominal syndrome. (Letter) Am J Med Genet 1996;61:401-2.

13. Engun SA. Embryology, sternal clefts, ectopia cordis, and Cantrell's pentalogy. Sem Pediat Surgery 2008;17:154-60.

14. Genest DR, Di Salvo D, Rosenblatt MJ, Holmes LB.Terminal transverse limb defects with tethering and omphalocele in a 17 week fetus following first trimester misoprostol exposure. Clin Dysmorphol 1999;8(1):53-8.

15. Emanuel PG, García GI, Angtuaco TL. Prenatal detection of anterior abdominal wall defects with US. Radiopgraphics 1995;15:517-30.

16. Moncada I, Ortiz A, Velásquez O, Moncada C. Pentalogia de Cantrell. Reporte de un caso. MedULA 2005;13:33-7. 